

\section{Museos y coyuntura de principios del siglo XXI en Bolivia ${ }^{1}$}

\section{Artículo de investigación}

Recibido: 21 de agosto de 2018

Aprobado: 30 de septiembre de 2018

\section{Javier Reynaldo Romero Flores}

Universidad Mayor de San Simón, Cochabamba, Bolivia

ja.romero@umss.edu.bo

Cómo citar este artículo: Romero Flores, Javier Reynaldo (2019). Museos y coyuntura de principios del siglo XXI en Bolivia. Estudios Artísticos: revista de investigación creadora,5(6) pp. 44-59.

DOI: https://doi.org/10.14483/25009311.14102

\section{(c) (i)}

https://creativecommons.org/licenses/by/4.0/deed.es

$<$

Museo de Arqueología, La Paz Bolivia. Foto: JoAnn Miller. Usuario Flickr.

\section{Resumen}

A partir de una corta revisión del museo y su transformación en el tiempo, se hace referencia a la importancia de la política en su administración. Luego, en función de la dinámica global y la disputa entre el Estado y el Mercado por la posesión de los bienes patrimoniales, que son resguardados en los museos, se reflexiona acerca de los conflictos, las tensiones y algunas salidas que se están dando en el plano global. A partir de este contexto, de manera breve, se reflexiona sobre el estado actual y el rol de los museos en la sociedad boliviana de cara al siglo XXI.

\section{Palabras claves}

Museo; gestión patrimonial; políticas culturales; Bolivia

\section{Museums and the juncture at the beginning of the XXI century in Bolivia}

\footnotetext{
Abstract

After a short review of the museum and its transformation over time, the article tackles the importance of politics in its administration. Then, we reflect on the conflicts, tensions and some openings that are taking place at the global level, stemming from complex dynamics and the dispute between the State and the Market for the possession of heritage assets, which are sheltered in museums. From this context, we offer a brief reflection on the current state of museums in Bolivian society and on their role for the twenty-first century.
} 


\section{Keywords}

Museum; heritage management; cultural policies;

Bolivia

\section{Les musées et la conjoncture du début du XXle siècle en Bolivie}

\section{Résumé}

Après une brève revue du musée et de sa transformation dans le temps, l'article aborde l'importance de la politique dans son administration. Nous nous pencherons ensuite sur les conflits, les tensions et certaines ouvertures qui se produisent au niveau mondial, résultant de dynamiques complexes et du conflit entre l'État et le Marché pour la possession de biens patrimoniaux, qui sont abrités dans des musées. À partir de ce contexte, nous proposons une brève réflexion sur l'état actuel des musées dans la société bolivienne et sur leur rôle pour le XXle siècle.

\section{Mots-clés}

Musée ; gestion du patrimoine ; politiques culturelles; Bolivie

\section{Museus e a conjuntura do início do século XXI na Bolívia}

\section{Resumo}

Após uma breve revisão do museu e sua transformação ao longo do tempo, o artigo aborda a importância da política em sua administração. Em seguida, refletimos sobre os conflitos, tensões e algumas aberturas que estão ocorrendo a nível global, decorrentes de dinâmicas complexas e da disputa entre Estado e Mercado pela posse de bens patrimoniais, que são abrigados em museus. A partir desse contexto, oferecemos uma breve reflexão sobre o estado atual dos museus na sociedade boliviana e sobre seu papel para o século XXI.

\section{Palavras-chaves}

Museu; gestão do patrimônio; políticas culturais; Bolívia

\section{Kawachiikuna tiaska sug luarpi kai tinpupi kagta kai atun wata kaskasina iskai chungapi sugwa kai alpa Bolviamanda}

\section{Maillallachiska}

Ña kaiaringapa chi kawachi imasa kamba ruraska tiaska i imasami tiimpu lialimi manunaia, chasa iapasuma uiakuna tia kai suma iukaskapi. Nispa kaiarimi sugma sugma rimanakui chi maipimi wakachiska iukankuna i chasaiatata rimankuna imamiiukankuna parpura chi atun wasapi musui suti luarpi, kaipipas ugnarinkuna iapa chi iapa iakichi tiauramanda, chasaitatami uigsinkuna kawangapa atun kawaima.ña kai kawaskauramanda chaiami ashia iuiarii tiami imasami kunaura kausai tia kai alpakunapi Bbolivia alpa sutipi kai iskai chunga sug siglo kaura.

\section{Rimangapa Ministidukuna}

Sug wasi kawangapa tiaska; pipa kagta; sug iuiai pangapi kilkaska iurakuna kai Bolivia suti alpapi

\section{Introducción}

Durante más de dos siglos el uso institucionalizado de los museos ha transitado entre dos extremos, que poco a poco, con el tiempo, se ha ido flexibilizando desde algunas propuestas intermedias. El sentido inicial de origen, que acompaña al "museiom" (Egipto) o al "museum" (Grecia) está relacionado con la idea de "templo", conectada con la "adoración" del arte y el conocimiento. Este sentido, con sus transformaciones, surge en Egipto y Grecia y llega hasta el auge del poder eclesial del siglo XVI en Europa.

Con el surgimiento del capitalismo, la concepción de este espacio y su actividad, empieza a cambiar de sentido. De aquella idea extrema de templo se transita hacia otra, también extrema, la que hace énfasis en el modo en el que se muestran los objetos, a manera de "centro comercial". Esta idea, se va imponiendo poco a poco, sobre todo en los Estados Unidos, hasta convertirse en la corriente

1 Una versión previa de este artículo fue presentada como ponencia en el Primer Encuentro Internacional de Museos. La Paz (Bolivia), agosto de 2018 
dominante de fines del siglo XX. Así, el Mercado y su lógica celebran un triunfo temporal.

Estas concepciones, antagónicas, la de "templo" y la de "centro comercial", son la consecuencia de proyectos políticos a partir de los cuales se ha instrumentalizado la administración de determinadas colecciones o archivos. Sin embargo, otro tipo de proyectos, desarrollados desde una racionalidad histórica, que relativizan ambos extremos, aportan también a conciliar aquellas tensiones. Sin embargo, el siglo XXI y el surgimiento de nuevos centros de poder económico está proyectando el resurgimiento del rol del Estado en la construcción y mantenimiento de los museos.

Si partimos de una concepción de museo, que no se quede en la idea de repositorio, que se proyecte como dispositivo para la activación de la memoria - es decir, como un espacio que guarda información y que puede proyectar horizontes de sentido que han sido bloqueados, vaciados o manipulados por las estructuras de poder dominantes-entonces, el museo, como la cultura, se convierten en un campo de conflicto de representaciones, en un campo de batalla en el que las ideologías entran en disputa.

Lo anterior no puede ser comprendido en su real magnitud, si no se parte de concepciones claras de cultura y museo. Y en un mundo tan complejo, en el que la geopolítica global de occidente ha tomado a la cultura, desde hace varios siglos, como uno más de sus espacios para operar; los sujetos y la posibilidad de construcción de proyectos políticos insurgentes deberían tomar en cuenta la importancia de la recomposición de la idea de cultura, como campo de batalla. En este contexto, ya no estaría encubierto el rol del museo, como espacio de encuentro, pero al mismo tiempo como lugar de disputa de representaciones. Solo de este modo se podrá detectar y hacer visible procesos de dominación, por una parte y, por otra, la instalación y la apertura hacia procesos de liberación.

En este sentido, en el actual contexto global en el que en Europa y los Estados Unidos la lógica del Mercado sigue presente y cada vez con más fuerza y algunos países arabes y asiáticos recuperan patrimonio e invierten fondos estatales millonarios, para construir museos de última generación orientando procesos que Mignolo (2015) los ha denominado desoccidentalización. ${ }^{2}$ Nos preguntamos: ¿Cuál es el horizonte de sentido real de la política cultural boliviana y cómo influye en el estado actual de los museos?

Cuando hacemos referencia a la política cultural nos referimos a normativas institucionalizadas que puedan regular los procesos que producen y reproducen cultura. Es decir, nos estamos refiriendo a la producción, circulación y consumo de representaciones culturales y a su consecuencia, la reproducción de prácticas culturales. Estos procesos complejos son los que determinan la hegemonía, predominancia o ausencia de determinadas prácticas culturales y de este modo los que controlan estos procesos, es decir, las estructuras de poder imponen como la dominante. Esto se da en el complejo campo de batalla ideológico, que estamos denominando cultura y es de este modo que se reproduce o se transforma la subjetividad de la población. Por esto pensamos que un proyecto político que está interesado en transformar la subjetividad de su población, debe priorizar las políticas culturales.

Nuestra corta reflexión la hemos desarrollado en tres subtítulos. En el primer subtítulo, de manera breve mostraremos el surgimiento de las nociones de museo y sus posteriores transformaciones. En el segundo subtítulo nos detenemos para mostrar la importancia política en la administración de los museos y cómo esta ha servido para orientar

\footnotetext{
2 En Mignolo (2015) encontramos la diferencia entre dos tipos de contradicciones, entre las que se debate el mundo actual. La primera entre occidentalización y desoccidentalización y la segunda entre colonización y descolonización. En el primer caso, al proceso de difusión global de la modernidad, su modo de producción dominante, el capitalismo y una historia en la que la cultura europea es el canon a ser imitado por todas las culturas del planeta; se opone un proceso en el que se mantienen los patrones que tienen que ver con el capitalismo y la modernidad, pero se re-significa la historia y el canon occidental de cultura se sitúa en el momento anterior a las hegemonías locales. Esto sucede en las nuevas economías emergentes de los países árabes y algunos del Asia. Mientras que la segunda contradicción, lo que se opone a la difusión global de la modernidad, como colonizadora del resto de los países del mundo, son procesos en los que se intenta superar la modernidad y el capitalismo desde horizontes políticos locales, alternativos a la modernidad y al capitalismo.
} 


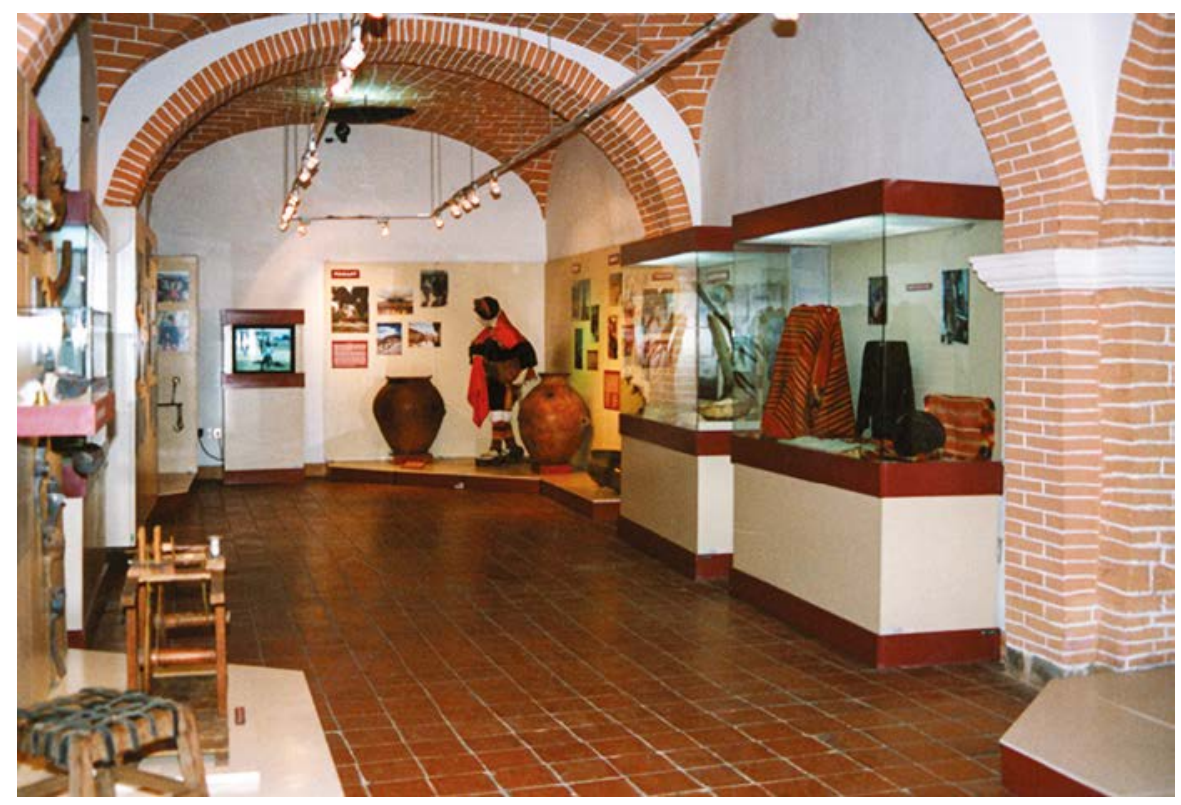

Imagen 1. Sala Tarabuco. Museo Nacional de Etnografía y Folklore (La Paz, Bolivia).

Foto: Javier Romero (2006).

proyectos que son consecuencia de ideologías distintas y contradictorias. Finalmente, en el tercer subtítulo, realizamos una reflexión preliminar sobre el estado actual de algunos museos en Bolivia.

\section{Las concepciones de museo y su transformación en el tiempo}

No es novedad que la dinámica en la producción, circulación, consumo y transformación de representaciones y prácticas culturales, se haya transformado enormemente en el transcurso de los últimos siglos. Del mismo modo, la concepción, la organización, el funcionamiento y uso de los museos, también ha sido parte de aquellas transformaciones. Estos procesos, que producen, pero al mismo tiempo son parte de, consecuencias diversas, han sido asimilados y abordados por los museos de diferente modo, dependiendo el contexto económico-político global, regional y local.

Son aquellas transformaciones y sus consecuencias, a veces no tan visibles pero sobretodo, muy poco atendidas desde la gestión política en nuestro país, las que nos obligan a hacer algunas preguntas: ¿Cómo surge y cómo se han dado los cambios en la concepción del museo con el transcurso del tiempo y cómo se han recibido estos cambios en Bolivia? Para responder, realizaremos un repaso rápido y breve de algunos hitos en la transformación de los museos y luego, en la parte final, intentaremos comprender su repercusión en nuestro país.

Se sabe que en las culturas africanas, las asiáticas, las de la Mesopotamia y el Antiguo Egipto, así como los Mayas y los Aztecas, incluso en Tihuanacu y el Tawantinsuyu, fueron producidos objetos suntuosos y muchos de ellos también fueron utilizados para ser expuestos en ocasiones especiales, por ejemplo en algunos rituales festivos, o en enterramientos y monumentos funerarios.

Sin embargo, ha sido sobre todo en los países europeos donde se ha cultivado un modo particular de relacionamiento con los objetos. $Y$, aunque este es un fenómeno relativamente reciente, desarrollado, discutido y abordado en estos países, el proceso de colonización global de la Modernidad eurocéntrica ha hecho que la constitución de la idea de museo y sus transformaciones, sean tomadas en cuenta en todos nuestros países. 
Aquella diferencia en el relacionamiento con los objetos, surgida en Europa y que produce el surgimiento del museo como lo conocemos actualmente, tiene que ver con la producción de un tipo de racionalidad que logró separar al sujeto del objeto, la naturaleza de la cultura y con esto logró constituir, para el siglo XX, lo que los filósofos han denominado el paradigma de la consciencia. ${ }^{3}$ Se trata de un modo de relacionamiento con la realidad caracterizado por dos componentes: un sujeto, productor de conocimiento a través del uso exclusivo de la razón, y un objeto resultado del proceso de construcción de conocimiento y carente de razón.

Este proceso se ha desarrollado de distinto modo en otras culturas, en las que los objetos están cargados de funciones prácticas o simbólicas y el relacionamiento con estos incluye además de la razón, la fe y la sensibilidad. ${ }^{4}$ Sin embargo, en Europa, desde el surgimiento del capitalismo la producción de algunos objetos, además de la función contemplativa, cobró valor monetario específico y en la actualidad sirven para generar riqueza. Es decir, el proceso de objetualización de la realidad empezó a relacionarse con otro, el de la mercantilización.

Los cambios que se han venido dando en el museo están relacionados con todas estas transformaciones $y$, al mismo tiempo, este espacio se ha hecho parte también de ellas. Veamos cómo se inicia este proceso. Como ya se dijo, los antiguos enterramientos, las pirámides egipcias, por ejemplo, mantenían un orden en su interior y cuidaban un tipo de organización de los objetos que respondía a ciertos criterios y jerarquías en el espacio. Lo mismo sucedia en otras partes, en el Asia o en el antiguo México y en las culturas de los Andes. Sin embargo, en la antigüedad, este tipo de espacio no era denominado como tal. Se sabe que en Egipto existían espacios que hacian referencia a

\footnotetext{
3 El paradigma de la conciencia es una forma de relacionamiento con la realidad desde la cual se ha producido un marco conceptual. Es parte de la filosofía clásica. Se trata de la idea de un sujeto autárquico, autónomo, reflexivo que posee la "capacidad" de la conciencia de sí. Este, desde su pensamiento regula todo en el mundo y se relaciona con la realidad de un modo objetualizado, es decir como sujeto-objeto. Este tema puede ser ampliado en Apel (1991) y Apel, Dussel (2005). 4 Este tema puede ser ampliado en Panikkar (2009).
}

un tipo de templo, que no era ofrendado a deidades, sino más bien al conocimiento. Se trataba de un espacio que estaba designado al estudio, una especie de universidad.

La importancia del desarrollo egipcio en aquella época se complementó con la expansión griega. De este modo se produjo la creación de la Biblioteca de Alejandría que contenía todo el conocimiento y desarrollo acumulado por los egipcios. Esta fue creada bajo el poder de Alejandro Magno, cuando Egipto empezaba a declinar. Sin embargo a este espacio también se lo ha denominado Museo de Alejandría. Se trataba de un espacio para la investigación que, además de contar con una importante biblioteca, tenía diferentes salas para discusión y otros espacios especializados (Estrugas, 2005).

El término museion, que hace referencia al culto a las musas en la antigua Grecia, fue reemplazado posteriormente por el término latino museum, en el momento del despliegue del Imperio Romano. Este se refería también a un lugar que servía para el desarrollo de discusiones filosóficas y, como en los casos anteriores, servía también como espacio en el que se guardaban piezas raras de épocas anteriores y de lugares alejados. Pero, este tipo de espacio también estaba relacionado con prácticas de música, poesía y otras expresiones a las que luego se vino a denominar artes.

Posteriormente, mientras otros proyectos civilizatorios en el planeta eran parte de sistemas interregionales particulares, el Maya-Azteca, el Inka, el Chino, el Hindú fueron desarrollando sus propios procesos civilizatorios, entre los siglos $\mathrm{V}$ y $X V_{1}^{5}$ se fue configurando la articulación entre la Mesopotamia y el África a través de Egipto. Esta articulación y la conexión, a través del mar Mediterráneo, dio lugar a un nuevo proyecto civilizatorio articulado como Modernidad y, desde este, se constituirá por primera vez un sistema mundo, colonizado y globalizado desde Europa (Dussel, 2000). La historia construida desde aquel continente denominará el lapso de tiempo entre estos siglos con el nombre de Edad Media.

$5 \quad$ Este proceso es explicado en Dussel (2000). 
En este contexto, aquella región transitará del esclavismo, al feudalismo y luego, cuando desde Europa se difunda su proyecto de colonización global, el modo de producción capitalista se convertirá en dominante. Sin embargo, hasta el siglo $X V$ se desarrollará una serie de acontecimientos en diferentes ámbitos, que generarán un escenario a partir del cual en el Renacimiento se hable del museo como un lugar destinado a colecciones privadas de objetos.

\footnotetext{
... las invasiones bárbaras eran una constante amenaza; las antagonías entre los señores feudales, los ideales caballerescos, la ignorancia, el pillaje, la rapiña y, en consecuencia, el hambre, la insalubridad y las altas tasas de mortalidad definen de manera muy general las realidades que vivió la sociedad medieval. La iglesia católica se expandió como dogma e institución por toda Europa. El poder acumulado por la iglesia durante este período le permitió hacerse custodio y guardián del legado de la cultura pagana-griega y romana. La abadía primero y la catedral después, se convirtieron en el centro de la actividad económica, educativa y artística. La clase noble no sólo confiaba la educación y formación de sus miembros a la iglesia, sino que le cedió también importantes objetos de valor artístico e histórico que habían pertenecido a sus antepasados. Se inició así el coleccionismo de arte en el seno de la institución eclesial, y que perdura hasta nuestros días (Linares, 2008, p. 2).
}

Las circunstancias se habían dado para que la iglesia y la nobleza europea se convirtieran en los "custodios" de obras de arte y objetos antiguos locales y de otras regiones. Años más tarde, desde el sentido histórico construido y destacando a Grecia y a Roma, se construirá una idea de "origen" de Europa y su desarrollo, como referente global. Durante varios siglos los templos católicos funcionaron evocando al futuro museo. Las pinturas y esculturas en su interior fueron encargadas a los mejores pintores y escultores de la época, para observar la mejor producción arquitectónica, pictórica, escultórica y musical. Era un tiempo en el que había que estar en los templos de las capitales europeas para apreciar aquellas obras.

De este modo, el momento del Renacimiento europeo marcará una nueva etapa en la música, la pintura, la escultura, la literatura y el conocimiento. Para el caso de las colecciones de objetos, el rol del erudito se convertirá en fundamental. También en esta época el mecenazgo servirá como uno de los pilares para que las mencionadas prácticas se desplegaran en aquellos países. Así, junto con lo anterior, se dio lugar a la formación de las primeras grandes colecciones y al surgimiento de los coleccionistas. Italia fue el centro en aquella época, lugares como Florencia y Venecia, apellidos como los Médici, nombres como Leonardo, Miguel Ángel o Rafael, entre otros, dieron lugar a objetos, espacios y colecciones privadas que posteriormente pasaron a la custodia de los gobiernos.

En este proceso, el centro de gravedad en la construcción de la idea de museo se seguirá moviendo hacia el norte de Europa. En el año 1753 será fundado el Museo Británico en Londres y en el año 1791, luego de la Revolución Francesa, el Palacio del Louvre será destinado, mediante decreto, a que cumpla funciones "artísticas" y científicas, pero recién en el año 1793 será abierto al público.

Pintura, escultura, conocimiento, entre otras prácticas, fueron parte de un campo que estaba provocando especial atención por algunos sectores de la aristocracia y el poder. La llustración, por su parte, jerarquizó aún más aquellas prácticas relacionadas con la pintura, la escultura, la líteratura, pero sobre todo la historia. Por ello, no es un dato menor que en 1778 , antes de la Revolución Francesa, ya se contaba con un proyecto para una pinacoteca que estaría emplazada en su Gran Galería. Es decir, el proyecto del gran Museo del Louvre ya existía.

El cambio más importante que se dio con la Revolución Francesa, no fue el proyecto de museo como tal, más bien fue la posibilidad de acceso a aquellos objetos y con esto la posibilidad de acceso al conocimiento de la población que no era parte de la aristocracia. Con la Revolución, el pueblo francés tenía las mismas condiciones que la aristocracia para el acceso a las obras de arte y al conocimiento.

En todo lo expresado, constatamos que el acceso a objetos y documentos ha estado siempre relacionado con el poder político. Sin embargo existe una diferencia en el modo de organización de aquel poder en la antigüedad y el que se va dando a partir del Renacimiento y la llustración. Con estos dos acontecimientos, que tienen bastante complejidad, lo que sucede con el proceso formativo del museo es que se consolida su secularización, pero también la posibilidad de negociar con aquellos objetos. 
Posteriormente, con la Revolución Francesa y el surgimiento del Estado Moderno, la Iglesia Católica va cediendo su poder al nuevo Estado y este asume el poder absoluto sobre determinados bienes. En este caso específico, el nuevo Estado se hace cargo de los bienes patrimoniales de la nueva República, sobre los cuales se deberá construir la nueva idea de nación. De este modo el conocimiento y la propiedad de los objetos "patrimoniales" son fundamentales para la creación de un nuevo orden mundial surgido siglos atrás, pero consolidado con el surgimiento del Estado Moderno a fines del siglo XVIII.

\section{La importancia política en la administración de los museos}

Desde el surgimiento de la idea de museo varias cosas han cambiado en su configuración, sin embargo, lo que no se ha modificado ha sido su relación directa con las diferentes estructuras de poder. Como nos recuerda François Mairesse: “La historia del museo se superpone con la de reyes y Estados" (Mairesse, 2013, p. 104). Y esto no ha cambiado desde la creación del museo de Alejandría. En Grecia, en el Renacimiento y en la Modernidad, hasta el siglo XX, la razón de Estado ha sido la que ha guiado la estructura y organización del museo. Sin embargo, a fines de aquel siglo, algo de esto ha empezado a cambiar.

Es por esta razón que pensamos es fundamental hacernos otra pregunta. ¿Cuál es la importancia política en la relación que existe entre la administración del Estado y la administración de los museos? Entonces, la posibilidad de encontrar una respuesta a esta pregunta, pasa por comprender la dinámica del poder y el modo en el que este se legitima en la población.

Cuando surge el Estado Moderno a fines del siglo XVIII, con la Revolución Francesa, surge también la necesidad de construir un relato que pueda sustentar históricamente a la nueva República. Este proceso de imaginar una nueva comunidad, ${ }^{6}$ anteriormente inexistente, necesitaba de ciertos dispositivos que logren la construcción de este imaginario en la población que formaría parte de aquel

$6 \quad$ Este tema ha sido desarrollado por Anderson (1993). nuevo Estado. Uno de aquellos dispositivos es el museo moderno, que servirá como herramienta en la invención de una específica memoria histórica.

Posteriormente, en el siglo XIX se darán otros movimientos de independencia en Europa, Norte, Centro y Suramérica. Inmediatamente después, como parte de la institucionalización de los nuevos proyectos de nación, los gobiernos se hacen cargo de la creación y administración de nuevos museos. Sin embargo este no es un proceso aislado, va sobretodo de la mano de la organización de diferentes instancias de gobierno, entre las que los sistemas escolares son fundamentales.

Así, el museo se posiciona como uno de los nuevos símbolos del poder, templo laico cuyas imágenes [...] este último se asegura de difundir (y de controlar), mientras el museo se propaga por las provincias al mismo ritmo que la alcaldía, la escuela y la biblioteca. El siglo XIX, el siglo de los museos es ante todo el de las construcciones de provincia. Ya se han fundado casi todas las instituciones europeas más grandes; el movimiento se extiende, pues, en cada jefatura de departamento, canton, etc. Así, con el apoyo de ciudades y comunas, se crean establecimientos encargados de realzar el valor del pasado regional y las riquezas patrimoniales (Mairesse, 2013, p. 109).

De este modo, en Europa, el museo es incorporado como un componente más del nuevo proyecto político de la nación moderna y su rol fundamental es la formación, como parte del sistema de educación no formal, del nuevo sujeto moderno. Sin embargo, los procesos de construcción de las naciones no fueron homogéneos, al contrario, estos se desarrollaron de manera heterogénea. La actual institucionalidad de algunos Estados, los menos, ligada a su poder económico-políico global, a diferencia de cierta carencia de institucionalidad y la poca relevancia económica y política de otros, son actualmente uno de los indicadores en la diferencia de estos procesos globales, que están marcados por la colonialidad del poder, del ser, del saber y de la Naturaleza, como afirman los argumentos de Dussel (2003), Quijano (2003), CastroGómez (2003) y Lander (2003), entre otros.

El Renacimiento y la llustración, que tuvieron como su centro de acción algunos países de Europa, están relacionados con procesos de dominación colonial hacia el resto de países del mundo. Además, aquellos procesos fueron los que insidieron directamente en las posibilidades políticas de 
construcción de conocimiento, luego de instalar y expandir por todo el planeta el proyecto de globalización de la modernidad eurocéntrica. Este tenía directa relación con la construcción de las teorías dominantes, como afirma De Sousa Santos, cuando referencia que: "la teoría crítica eurocéntrica fue construida en unos pocos países europeos (Alemania, Inglaterra, Francia, Rusia e Italia) con el objetivo de influenciar las luchas progresistas en esa región del mundo (De Souza, 2010, p. 18). En este complejo proceso se dio, además, el surgimiento del capitalismo, que tendrá consecuencias importantes en la transformación de la administración de los museos a fines del siglo XX.

Del mismo modo, es importante remarcar que la idea de museo es parte de aquella construcción colonial instalada desde el poder, orientada hacia el ser (europeo) y relacionada con el saber (construido en Europa), que se ocupa de producir una historia victoriosa de occidente. En el proceso de construcción de esta historia se imponen, con mucha fuerza, las ideas de Modernidad y Tradición. Estas funcionarán como dos componentes de un mismo proceso, en el que la Modernidad, como dominación, es la referencia del ser, de Europa y, la
Tradición, derrotada por la dominación moderna, el reflejo de lo arcaico, del resto del mundo que mira a Europa como el modelo a seguir, o de lo que este continente ya no quiere ser.

La composición del museo moderno juega con esta construcción y la instala en la narrativa de sus exposiciones. "Cuando se piensa en diversos museos del mundo occidental, como el Museo Británico, podría sostenerse que existe o bien una "tradición" europea (la Antigüedad griega y la Edad Media latina, por ejemplo) o bien muchas "tradiciones originarias de sociedades no europeas que a menudo son vistas - $y$ descritas - desde perspectivas europeas" (Mignolo, 2015, p. 138).

Entonces, el museo, respaldado, financiado y administrado desde el Estado, como parte de los dispositivos de producción y reproducción de su propio proyecto político, se consolida en Europa, junto a los países que se disputan el poder y la hegemonía global. En el siglo XX, luego de la denominada Segunda Guerra Mundial, Estados Unidos, no solo entra en esta disputa sino que se consolida como la nueva potencia global y, con esta influencia, se iniciará el momento de un mundo bipolar. Dos

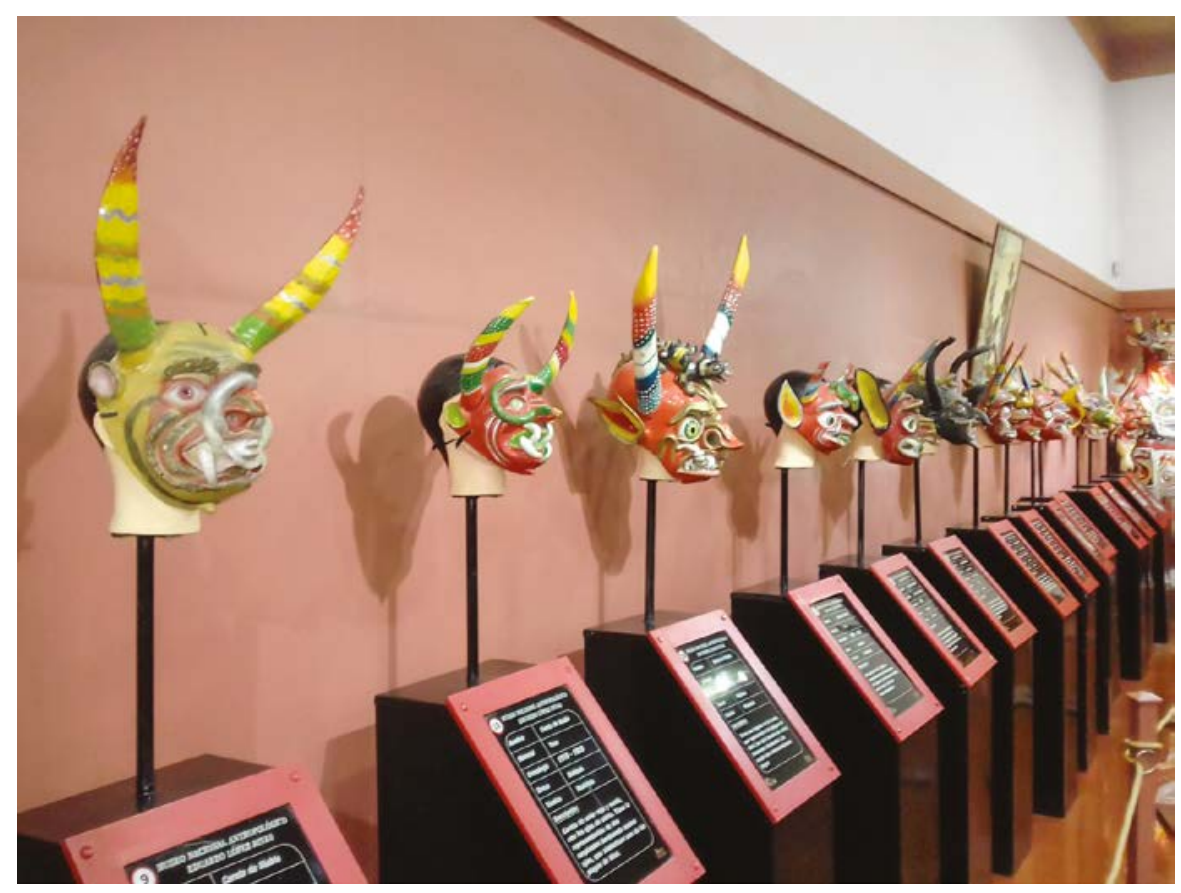

Imagen 2. Máscaras danza Diablada. Museo Antropológico (Oruro, Bolivia). Foto: Javier Romero (2018). 
modos de producción posibles entran en disputa y se hacen parte de proyectos políticos que se mueven polarizados, entre la posibilidad de regulación de la economía desde las relaciones de Mercado, o desde los criterios de un Estado protector. La primera, es parte del proyecto Liberal y la segunda se plantea como Capitalismo de Estado y es el momento de transición hacia el socialismo.

Los museos no son ajenos a estas disputas. La tendencia en los Estados Unidos es abrirlos hacia el comercio libre y desde esta lógica regulan su administración y su concepción. Mientras los gobiernos eurpeos son más reticentes al Libre Mercado. Sin embargo, coincidentemente, en los países europeos en los que prosperó el capitalismo también fueron prosperando los gabinetes de curiosidades y las galerías de arte. Fue también en este contexto en el que los grandes museos realizaron alianzas con familias de grandes fortunas.

Si bien, en el siglo XIX fueron exitosos los museos industriales que servían de incentivo para la invención de nuevas maquinarias, posteriormente perdieron interés. En la misma época surgieron los museos comerciales, que servían para promocionar productos para la exportación. Estos últimos funcionaron un corto tiempo como ahora lo hacen las grandes ferias de exposición. Durante el tránsito al siglo XX y en parte de la primera mitad los investigadores ligados a los museos migrarán a los espacios académicos y poco a poco las universidades cobrarán más relevancia en el campo de la investigación.

Posteriormente, en la segunda década del siglo XX, la administración estatal de los museos declina, en favor de fondos privados. Incluso, los presupuestos de los grandes museos de Europa son recortados. Esto recrudece en 1989 con la caida del Muro de Berlín y el surgimiento de un mundo unipolar influye en el despliegue global de un neoliberalismo extremo, que despoja a la mayoría de los Estados de sus responsabilidades y los museos no son la excepción. La mercantilización extrema con la idea del libre mercado se fue apoderando, no solo de los espacios comerciales, sino también, de la subjetividad de la mayoría de la población en el mundo.

Este es un tiempo en el que se consolida el proyecto político liberal, en el que se instala la economía de mercado para liberar de responsabilidades al Estado. Sin embargo, lo que se encubre en esta coyuntura es que los grandes capitales transnacionales han sido organizados en coorporaciones transnacionales y sus grandes ejecutivos son también actores políticos que inclinan los beneficios de aquellos recursos hacia la ganancia de sus empresas y no hacia las poblaciones pobres de los países. En la mayoría de los países la administración estatal de los recursos naturales, comprendidos como materias primas para la producción industrial, está dirigida al beneficio de aquellas coorporaciones. En esta época, de modo constestatario, surgen también propuestas de museos comunitarios y los "eco-museos", que son espacios impulsados por comunidades locales.

Es en este contexto, de hegemonía liberal y repliegue en la administración estatal que se llega a fines del siglo XX. Un naciente mundo multipolar con los países del BRICS se encargará de relativizar aquella hegemonía en el tránsito de siglo y nuevas y antiguas lógicas circularán por los museos. Se hace evidente que "Europa y Estados Unidos no necesitan gastar ingentes cantidades de dinero para construir sus identidades civilizatorias, porque ya lo hicieron. Europa empezó a hacerlo en el Renacimiento; Estados Unidos, desde comienzos del siglo XX" (Mignolo, 2015, p. 142).

La industria, que fortaleció a la Modernidad por varios siglos, en su demanda de energía, irá produciendo nuevas posibilidades de riqueza, en los países árabes (productores de petróleo). Con esto, nuevas necesidades de patrimonialización fueron surgiendo en el mundo. El Museo de Arte Islámico de Doha, el Museo Nacional de Qatar, el Louvre de Abu Dhabi, son ejemplos de la importancia de los museos en los proyectos políticos de cada región. Por otra parte, la dinamización de las economías en el Asia también hará lo suyo, citamos el Museo de las Civilizaciones Asiáticas de Singapur y la Sucursal del Sur del Museo Nacional del Palacio en Taiwán.

Sobre estos procesos complejos y sus tensiones, Mignolo (2015) nos menciona diferentes trayectorias en relación a los museos, las prácticas artísticas y su gestión. Estas se mueven entre occidentalización y desoccidentalización o también entre, colonialidad y descolonización. Pero estas trayectorias están relacionadas con determinados proyectos políticos 
y tienen que ver con diferentes posibilidades de transformación, evidentes en este nuevo siglo.

Las nuevas regiones emergentes están produciendo descentramientos y desplazamientos culturales. Los grandes museos, en los países hegemónicos, activaron dispositivos para la dominación y las grandes cantidades de reliquias que robaron de países en todo el mundo siguen expuestas completamente descontextualizadas porque su proyecto hacía énfasis en evitar la activación de la memoria.

Mientras que los museos mencionados en Qatar, en Abu Dhabi o los de Asia están produciendo un giro en la geopolítica de la cultura y están generando desplazamientos ideológicos y culturales. En este caso, están orientados para servir como dispositivos para activar la memoria y se están haciendo parte de otros movimientos más complejos de desoccidentalización. Entonces, podemos decir que "lo que está sucediendo no es simplemente una imitación de la occidentalización, sino la puesta en práctica de una desoccidentalización, pues los estándares culturales occidentales están siendo apropiados y adaptados a sensibilidades, necesidades y visiones locales o regionales" (Mignolo, 2015).

En el ámbito de las civilizaciones y los museos, esto es una novedad significativa. Es decir, aquel proyecto político no tiene previsto abandonar la lógica del mercado capitalista, pero se ha propuesto un trabajo particular en la transformación de las representaciones culturales occidentalizadas para producir su desoccidentalización, es decir se ha iniciado un proceso para cambiar el orden histórico vigente. Si bien en el siglo XX Europa era el presente civilizado y el resto de las culturas eran el pasado por civilizar, esta transformación intenta situar a Europa en un estadio anterior al auge de los países árabes del siglo XXI.

De este modo en este panorama confuso en el que algunos Estados ceden el poder sobre los museos a fundaciones privadas, o los rentan para actividades familiares o comerciales; en los que la "marca museo" se negocia durante el transcurso de cuatro gobiernos en Francia a los países árabes, los pequeños museos declinan y desaparecen y nuevos museos surgen con la protección de Estados o princesas, la aparente derrota del Estado por el monopolio del Mercado no es tal y la disputa política entre occidentalización y desoccidentalización o colonialidad y descolonización, que se hace evidente en las prácticas culturales y la administración de los museos todavía tiene muchas cosas por decirnos.

Como vemos, el contexto es bastante complejo. Mientras los museos clasicos recortan presupuesto, 32 millones de dólares anuales para el Museum of Modern Art de Nueva York o 30 millones para el Metropolitan Museum en Londres, el Museo de Arte Islámico de Doha tiene 1.000 millones de dólares al año. Por otra parte el Gobierno francés cobra 1.000 millones de euros para ceder el nombre al museo denominado "El Louvre de Abu Dhabi" por 30 años y 6 meses, pero además se invierten muchos miles de millones más en construcciones extraordinarias y únicas en varios lugares del mundo, por ejemplo la Sucursal del Sur del Museo Nacional del Palacio en Taiwán costó 345, 24 millones de dólares.

Otro componente en el proceso de transformación en la administración de los museos, surgido por la política de recortes presupuestarios, es el alquiler para actividades extra museo. Estas actividades tienen como único fin cubrir fondos que han sido recortados con las políticas administrativas recientes. Por ejemplo, no existe información sobre el precio que se pagó al Louvre de París por el rodaje del video de Beyonce y Jay-Z, en este año. La información más cercana es del año 2015 y se dice que por el rodaje de un día se cobró un máximo de 23.000 euros. Por otra parte, en España, en el Museo Nacional de Arte de Cataluña pagaron 200.000 euros para la realización de la boda de la hija del magnate indio Lakshmi Mittal (La Nación, 2008). Este es el contexto global en el que se puede visibilizar intereses de poder y disputa por el espacio de los museos y por lo que ellos representan. En este contexto el Estado y el Mercado se siguen disputando aquellos espacios.

\section{El estado actual de los museos en Bolivia}

En este complejo contexto global, queremos pensar también en la política y los museos en nuestro país. Para ello haremos referencia, de manera resumida al proceso relacionado con las políticas 
culturales y con la organización y administración de los museos en Bolivia. René Zavaleta (1986) es drástico, pero contundente, cuando menciona que las élites en Bolivia se limitaron a realizar una adminstración hacendal de Bolivia, es decir administraron nuestro país como si fuera su hacienda y lo hicieron para beneficio propio. ¿Qué significa esto en el contexto de la historia de los museos en Bolivia? Simplemente que: si la construcción de nación no estaba en los planes de las élites bolivianas, la construcción y el mantenimiento de museos que acompañen aquel proceso fallido, no tenía ningún sentido.

Seguramente, por esta característica también los temas culturales no son prioridad en la agenda política hasta nuestros días. Uno de los primeros documentos en el que se menciona la creación de museos es la circular del 15 de mayo de 1872 , donde se instruye la creación de museos (Olivares, 2013). Sin embargo en años anteriores, en 1846, se inauguró el primer Museo Público, en el segundo piso del actual Teatro Municipal, en La Paz. Posteriormente en 1919, se autoriza alquilar el Palacio Tiwanaku para el funcionamiento del Museo Nacional y Mineralógico, hasta que en 1922 Bautista Saavedra autoriza la compra de aquel inmueble, en el que recién desde 1960 se reinaugurará el nuevo museo, con el nombre de Museo Nacional de Arqueología. Actualmente se mantiene este nombre y este museo en aquel predio.

Sin embargo, ni los museos ni las políticas culturales fueron la prioridad. Durante el tránsito del siglo $\mathrm{XIX}$ al siglo $\mathrm{XX}$, fueron varios los acontecimientos que se vivieron en Bolivia y la necesidad de enfrentarlos y superarlos mantuvo a gobernantes y población asumiendo lo urgente y descuidando lo importante. Desde fines del siglo XIX se sucedieron una serie de conflictos, la Guerra del Pacífico primero, luego la Guerra Federal, posteriormente, entrando al siglo XX, la imposibilidad de superar las diferencias entre liberales y conservadores, para luego enfrentar en el conflicto bélico en el Chaco al enemigo, que no estaba en las filas del ejército oponente. Aquel estaba en la propia subjetividad de bolivianos y bolivianas y fue a partir de aquel acontecimiento que algo de aquel enemigo fue derrotado en 1952, en la denominada Revolución Nacional.
Por ello pensamos que la verdadera preocupación por los museos en Bolivia se da en la segunda década del siglo $X X$, pero más por iniciativas $\mathrm{Y}$ luchas personales que por decisiones políticas de los diferentes gobiernos de turno. Pensamos que el campo cultural siempre estuvo desatendido por las élites gobernantes, ni siquiera fue tomado en cuenta como un campo estanco y mucho menos como posibilidades de trayectorias y sentidos culturales.

Los temas relacionados con la cultura en Bolivia nunca superaron la confusión generada por la colonialidad del saber, la idea de arte como cultura, sirvió como dispositivo para la instalación de prácticas artísticas occidentales, como estatuto de cultura superior y esta confusión nunca fue resuelta. Por otra parte, la educación, entendida como instrucción y orientada hacia la occidentalización de la población, fue una prioridad, por encima de las políticas para las culturas. Por ello la existencia de varias leyes de educación y la carencia de una de culturas.

Aquel sistema de educación y sus reformas, pensado para la instrucción y destinado a la formación de empleados fieles y consumidores disciplinados del mercado capitalista primero y neoliberal después, ha servido para legarnos una historia de 193 años de racismo, de clasismo, de machismo, de homofobia, y de depredación de la naturaleza, como Bolivia. La educación en ningún momento ha sido pensada como producción, reproducción, consumo y circulación de representaciones culturales. Tampoco el museo ha tenido un rol de dinamizador cultural y menos como dispositivo que sirva para activar la memoria.

Sin embargo, luego de varios esfuerzos se inicia el apoyo estatal, pero sin una visión clara y orgánica por parte del Estado. Aquellas iniciativas y compromisos personales de gestión, en algunos cargos públicos, sirvieron para generar y sostener algunos museos $y$ archivos que transitaron con bastante incertidumbre gran parte del siglo XX. Posteriormente, el Archivo y Biblioteca Nacionales, la Casa de la Libertad, la Casa Nacional de Moneda y el Museo Nacional de Etnografía y Folklore pasaron a la tuición del Banco Central de Bolivia, hasta que en años posteriores se realizan gestiones para la creación de un ente descentralizado del Estado. 
En el año 1995, se crea la Fundación Cultural del Banco Central de Bolivia (FC-BCB), organismo que será administrado con fondos del Banco Central de Bolivia, pero que contará en su Consejo de Administración independiente conformado por personalidades relacionadas con el ámbito artístico y cultural. Esta nueva entidad irá transformando radicalmente la forma de custodia de las colecciones y archivos en Bolivia.

Sin embargo, es importante mencionar que este tránsito en la administración estatal orientada a espacios culturales no fue mérito del liderazgo político de aquellos gobiernos. Aquel proceso, de involucrar al Banco Central de Bolivia primero, como se venía haciendo en otros países, y luego de las iniciativas de creación de la FC-BCB, fue mérito de algunos gestores culturales, que produjeron las ideas y las fueron socializando entre algunas personas de la estructura de poder de aquel tiempo.

Por otra parte, es importante mencionar que aquel tránsito se dio en pleno auge del neoliberalismo $y$, en aquellas circunstancias, se quería que los centros culturales se conviertan en generadores de ganancia, o por lo menos que sean autosuficientes. Esto significaba orientar los objetivos hacia la generación de recursos, como se instruía desde las reuniones de consejeros de aquella época en la FC-BCB. En aquellas circunstancias fueron los directores de los centros que en varias ocasiones se pusieron al frente para impedir, por ejemplo, que no se cobren entradas a la población boliviana.

Pero, más allá de estos detalles, la característica de entidad descentralizada permitía, por lo menos en aquella época, ciertos niveles de independencia del gobierno central, en relación a las actividades y a los contenidos de estas. En aquel tiempo, fue el Museo Nacional de Etnografía y Folklore (MUSEF) el primer centro, con estas características, que recibió el respaldo del Estado boliviano; por ello es, en la historia de los museos en Bolivia, el primer museo que en la mayor parte de su extensión ha sido construido específicamente para esa actividad.

Este proceso, en la gestión estatal de los archivos y colecciones tuvo un momento de incertidumbre, con el exministro, Marko Machicao, cuando se planteó la posibilidad de que el Ministerio de Culturas recupere, como en décadas anteriores, la tutela de aquellos centros. Sin embargo esto no prosperó y actualmente la dinámica de aquella fundación no ha cambiado. Pero, si bien lo más relevante con este proceso es el resguardo de los archivos y colecciones, todavía quedan muchos pasos que dar a nivel de administración en la apertura de aquella información, contenida en archivos y colecciones, a pesar de los esfuerzos que se están haciendo.

A partir de la creación de la FC-BCB, se ha marcado una diferencia bastante visible de la valoración que se da a los procesos culturales en los diferentes ámbitos de administración estatal. La creación de esta entidad marca un antes y un después en la visión y acción de las actividades culturales. A diferencia del gobierno municipal de la ciudad de La $\mathrm{Paz}$, que ha puesto en vigencia una Ley Municipal de Culturas, meses atrás, la administración directa de los espacios culturales, sin una legislación específica de culturas es poco alentadora, ya sea en el gobierno central, en los gobiernos departamentales, de los municipios, incluso en universidades del sistema nacional el panorama es poco alentador. Se trata de espacios que no tienen presupuestos, no cuentan con laboratorios de conservación y el personal, por el tema salarial, no es especializado. A pesar de aquellas condiciones, en la mayoría de los centros, el personal es extremadamente comprometido con su trabajo.

Un último acontecimiento se da en el año 2017 que, por lo reciente en el tiempo, no podemos afirmar todavía si se trata de una transformación del modo en la administración de la cultura en el país, o de un hecho que sella la continuidad de lo mismo. Se trata de la inauguración del Museo de la Revolución Democrática y Cultural en Orinoca (Oruro). Esta es la primera obra monumental financiada directamente por el actual gobierno, según la prensa, se dice que costó aproximadamente 50 millones de bolivianos.

Una característica de este museo es que la producción de la idea, el diseño arquitectónico y su consolidación hasta la construcción, se da en un momento político que intenta superar la administración neoliberal del Estado. Es un momento en el que se intenta diferenciar el Nacionalismo Revolucionario del nuevo Estado y este se asume populista y plurinacional. Además, existe un mandato constitucional en el que la descolonización 
Imagen 3. Fachada 1. Museo de Orinoca (Oruro, Bolivia). Foto: Javier Romero (2017).

es un componente importante para la política de Estado, consecuentemente para sus instituciones. Entonces, ese sentido descolonizador debería ser parte de los contenidos conceptuales del nuevo Museo de Orinoca.

Es decir, aparentemente, salvando las diferencias de inversión de presupuesto con los países árabes, pero tomando en cuenta la inversión en cultura de los últimos años por este gobierno, parece un intento de recuperación de la tutela de los museos por parte del Estado y una apuesta de este para ingresar, finalmente, al campo de batalla de la cultura a través de la administración del nuevo museo. Sin embargo, la administración y sostenibilidad especializada de este museo, que debería acompañar la millonaria inversión todavía no es visible. Nuestra visita, en diciembre de 2017, nos dejó un panorama poco alentador sobre la sostenibilidad y proyección de aquel museo. Aunque todavía es prematuro emitir algún juicio, pensamos que si en las estructuras de gobierno no se da importancia

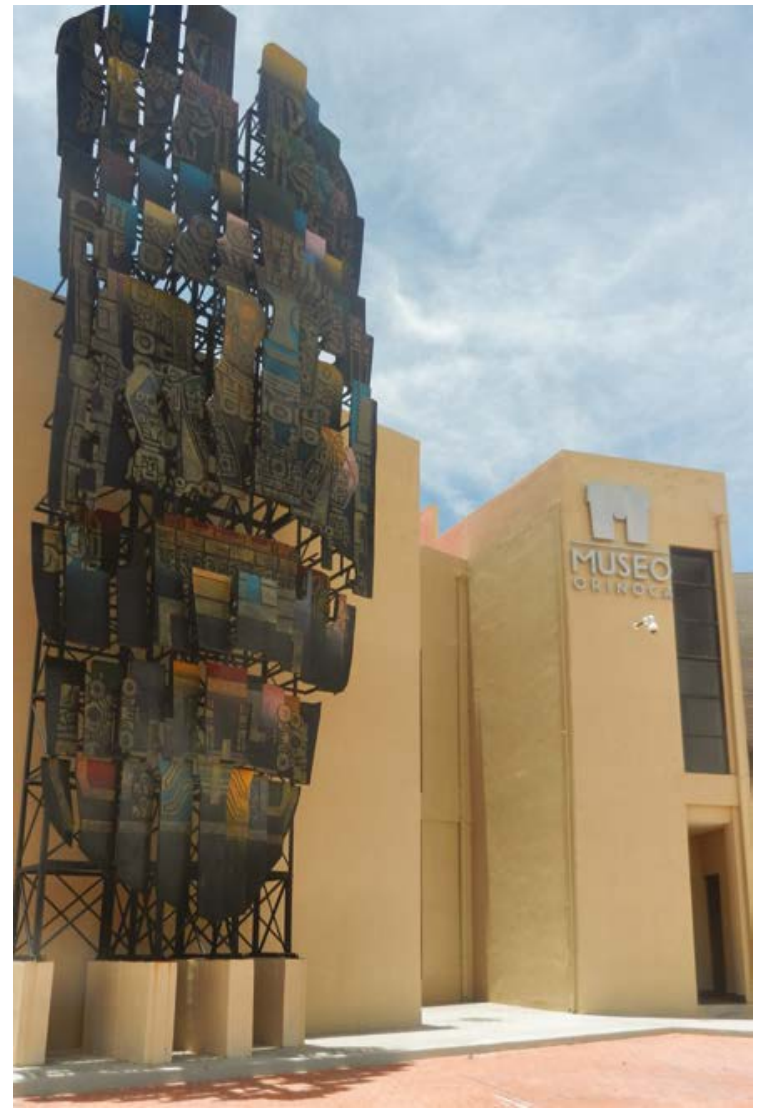

a los procesos de producción, circulación y consumo de representaciones culturales y su consecuencia la reproducción de prácticas culturales y con esto la constitución de subjetividades, la aparente reapropiación de los museos y archivos por parte del Estado quedará en un intento fallido que, como consecuencia, deja el campo cultural a expensas de la lógica del mercado capitalista moderno colonial.

\section{Conclusiones}

Cómo mencionamos al inicio, entendemos al museo como un dispositivo para la activación de la memoria. Sin embargo, para que esto sea factible en contextos como Bolivia, pensamos, es necesario desarrollar una doble tarea, que no solo es hacer accesible la historia y la cultura, que sería una de las tareas importantes; la otra, más compleja $y$, al mismo tiempo de alta urgencia, es desencubrir la historia y las culturas encubiertas por el proceso colonial. Esto significa re-componer y poner en 
circulación, con una alta exigencia de rigor, aquella información que por siglos de implementación del proyecto colonial y por la activación de sus dispositivos ha sido invisibilizada de diferentes modos.

Para lograr esta tarea, la segunda mencionada, es necesario aproximarnos a una comprensión de nuestro tiempo y los acontecimientos que se están produciendo. Esta deberá estar a la altura de aquellos acontecimientos. A partir de aquello, recién podremos comprender la magnitud de los nuevos retos que debemos enfrentar en el campo de la cultura y los museos. Preliminarmente, pensamos que una tarea importante es lograr la conformación de un sistema nacional de museos articulado y relacionado con otros sistemas, por ejemplo el de la educación o el de la producción artística. Este tipo de articulaciones son un tema pendiente, así como las transformaciones en la gestión cultural.

La misión de desencubrir, re-componer y poner en circulación representaciones y prácticas culturales encubiertas, como tarea, tiene que ver con un giro en el pensamiento de los administradores de cultura y museos. Este giro, que en realidad es un giro epistémico, recién hará visibles los nuevos retos para la cultura y los museos en Bolivia. Sin embargo, la tarea es titánica y estará impedida de ser realizada si, inicialmente y con la claridad de un proyecto político que se piense descolonizador, no se recompone el campo de la cultura, es decir, si no se desarrolla un nuevo trazado en aque campo y esto tiene directa relación con un entramado en el que lo legal, lo político y cultural se crucen y construyan puentes. Parte de este proceso es la necesidad de una ley de culturas, inexistente todavía en nuestro país.

Esta norma, además de producir puentes con lo jurídico y lo administrativo, deberá ser la norma reguladora de los procesos de enseñanza-aprendizaje y de transmisión de conocimientos ligados a la educación, formal y no-formal, pero también a las prácticas artísticas de todo el territorio nacional. Todo esto tomando en cuenta procesos tan complejos como son: la descolonización, la interculturalidad y la intraculturalidad en el entramado de las diversas culturas en Bolivia.

Lamentablemente, la actual gestión de la cultura está impregnada del economisismo del siglo XX y de la lógica del mercado, todavía vigente en las normativas actuales en nuestro país. Se piensa, cuando esto es posible, en inversión, pero no en sostenibilidad. Esto último se deja a la lógica y al movimiento del mercado. Aquí las ideas de turismo, folklore y patrimonio, que alimentan la mercantilización, la folklorización y la patrimonialización de la cultura, ${ }^{7}$ no solo interfieren los procesos de producción y circulación de representaciones y prácticas culturales, que son las que debería dinamizar aquella gestión, sino también vacían la posibilidad de un respaldo sostenido a la idea de educación y reproducción de valores.

Aquel giro epistémico, deberá también ayudarnos a acomprender que nos encontramos en un momento histórico, en el que se anuncia un cambio de época. Estamos en un momento en el que nuestras relaciones con la naturaleza deberán transformarse y transitar de un sistema de relaciones objetualizado y monetarizado, que entiende a la naturaleza como recurso natural, hacia otro, que nos sitúe en un modo de comprensión distinto, que sirva para evitar la destrucción de la vida de la naturaleza y de la humanidad.

Si bien esto significa transitar de la economía, rectora actual de la vida en el planeta, hacia la ecología. También debemos tener la suficiente claridad para darnos cuenta que se trata de una transformación cultural global de la que, como bolivianos, no podemos dejar de ser parte. Todo este panorama complejo tiene que ver con la cultura y la importancia de los museos, porque lo que también se juega en estos procesos es la imposición o la liberación de prácticas y representaciones culturales.

\section{Referencias}

Anderson, B. (1993). Comunidades Imaginadas. México: Fondo de Cultura Económica.

Castro-Gómez, S. (2003). "Ciencias sociales, violencia epistémica y el problema de la 'invención del otro". En La Colonialidad del saber: eurocentrismo y ciencias sociales. Perspectivas latinoamericanas. Edgardo Lander (Comp.). Buenos Aires, Argentina: CLACSO.

\footnotetext{
7 Estos tres dispositivos además de un cuarto, la demonización, han sido desarrollados en Romero (2017).
} 
De Sousa Santos, B. (2010). Descolonizar el saber, reinventar el poder. Montevideo, Uruguay: Trilce. Extensión universitaria. Universidad de la República.

Dussel, E. (2003). "Europa, modernidad y eurocentrismo". En La Colonialidad del saber: eurocentrismo y ciencias sociales. Perspectivas latinoamericanas. Edgardo Lander (Comp.). Buenos Aires, Argentina: CLACSO.

. (2000). Ética de la Liberación en la Edad de la Globalización y la Exclusión. Madrid, España: Trotta (3ra ed.).

Estrugas, G. (2005). “La biblioteca de Alejandría”. En TK 17 zk. ko abendua.

La Nación. (19 de junio, 2018). ¿Cuánto pagaron Beyonce y Jay Z por el Louvre? Recuperado de https://lanacionweb.com/farandula-y-espectaculos/ cuanto-pagaron-beyonce-y-jay-z-por-el-el-louvre/

Lander, E. (2003). "Ciencias sociales: saberes coloniales y eurocéntricos". En La Colonialidad del saber: eurocentrismo y ciencias sociales. Perspectivas latinoamericanas. Edgardo Lander. (Comp.). Buenos Aires, Argentina: CLACSO.

Linárez, P. J. (2008). El museo, la museología y la fuente de información museística. ACIMED [En línea], 17(4). Recuperado de <http://scieloprueba.sld.cu/scielo.php?script=sci_ arttext\&pid=S1024-94352008000400005\&lng=es\&nrm=iso > ISSN 1024-9435.

Mairesse, F. (2013). El museo híbrido. Buenos Aires, España: Paidós, Ariel.

Mignolo, W. (2015). Trayectorias de re-existencia: Ensayos en torno a la colonialidad/decolonialidad del saber, del sentir y del creer. Bogotá, Colombia: Universidad Francisco José de Caldas.
Olivares, F. (2013). Una mirada a la formación en el ámbito museístico boliviano, desde la gestión cultural. [en línea] http:// www.pedagogiademuseos.org/wpcontent/uploads/2013/12/ Olivarez_2013.pdf.

Panikkar, R. (2009). La puerta estrecha del conocimiento. Barcelona, España: Herder.

Quijano A. (2003). "Colonialidad del poder, eurocentrismo y América Latina". En La Colonialidad del saber: eurocentrismo y ciencias sociales. Perspectivas latinoamericanas, Edgardo Lander, (Comp.). Buenos Aires, Argentina: CLACSO.

Rojas-Sotelo, M. (2015). Compresiones: política cultural, economía y mercado. Estudios Artísticos, 1(1), pp. 56-81. DOI: https://doi.org/10.14483/25009311.10248

(2016). Tierra arrasada en la nación del branding. Estudios Artísticos, 2(2), pp. 32-55. DOI: https://doi. org/10.14483/25009311.11526

Romero, F. J. (2015). De la extirpación a la folklorización: a propósito del continuum colonial en el siglo XXI. Estudios Artísticos, 1(1), pp. 14-37. DOl: https://doi. org/10.14483/25009311.10246

Romero, J. (2017). Potencial político de lo festivo. Aprendiendo de la descolonización, Bogotá, Colombia: Universidad Distrital Francisco José de Caldas.

Zavaleta R. (1986). Lo nacional popular en Bolivia. México: Siglo XXI. 